\title{
A 2D simulation study of Langmuir, whistler, and cyclotron maser instabilities induced by an electron ring-beam distribution
}

\author{
AUTHOR(S): \\ Lee, K. H.; Omura, Y.; Lee, L. C.
}

\section{CITATION:}

Lee, K. H.... [et al]. A 2D simulation study of Langmuir, whistler, and cyclotron maser instabilities induced by an electron ring-beam distribution. PHYSICS OF PLASMAS 2011, 18(9): 092110.

\section{ISSUE DATE:}

2011-09

URL:

http://hdl.handle.net/2433/160674

\section{RIGHT:}

Copyright 2011 American Institute of Physics. This article may be downloaded for personal use only. Any other use requires prior permission of the author and the American Institute of Physics. The following article appeared in PHYSICS OF PLASMAS 18, 092110 (2011) and may be found at http://link.aip.org/link/?php/18/092110 


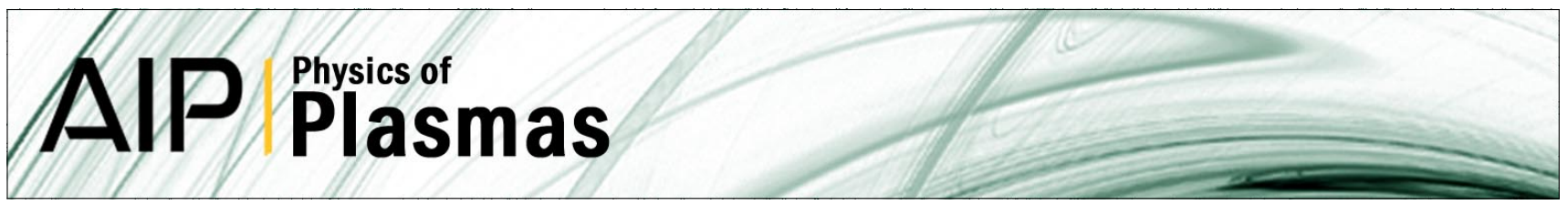

\section{A 2D simulation study of Langmuir, whistler, and cyclotron maser instabilities induced by an electron ring-beam distribution}

K. H. Lee, Y. Omura, and L. C. Lee

Citation: Phys. Plasmas 18, 092110 (2011); doi: 10.1063/1.3626562

View online: http://dx.doi.org/10.1063/1.3626562

View Table of Contents: http://pop.aip.org/resource/1/PHPAEN/v18/i9

Published by the American Institute of Physics.

\section{Related Articles}

Influence of equilibrium shear flow on peeling-ballooning instability and edge localized mode crash

Phys. Plasmas 19, 092503 (2012)

Electrostatic drift shocks and drift wave instability in inhomogeneous rotating electron-positron-ion plasmas Phys. Plasmas 19, 092102 (2012)

Relativistically modulational instability by strong Langmuir waves

Phys. Plasmas 19, 092101 (2012)

Simulation of filamentation instability of a current-carrying plasma by particle in cell method

Phys. Plasmas 19, 082119 (2012)

Rayleigh-Taylor-induced magnetic fields in laser-irradiated plastic foils

Phys. Plasmas 19, 082710 (2012)

\section{Additional information on Phys. Plasmas}

Journal Homepage: http://pop.aip.org/

Journal Information: http://pop.aip.org/about/about_the_journal

Top downloads: http://pop.aip.org/features/most_downloaded

Information for Authors: http://pop.aip.org/authors

\section{ADVERTISEMENT}

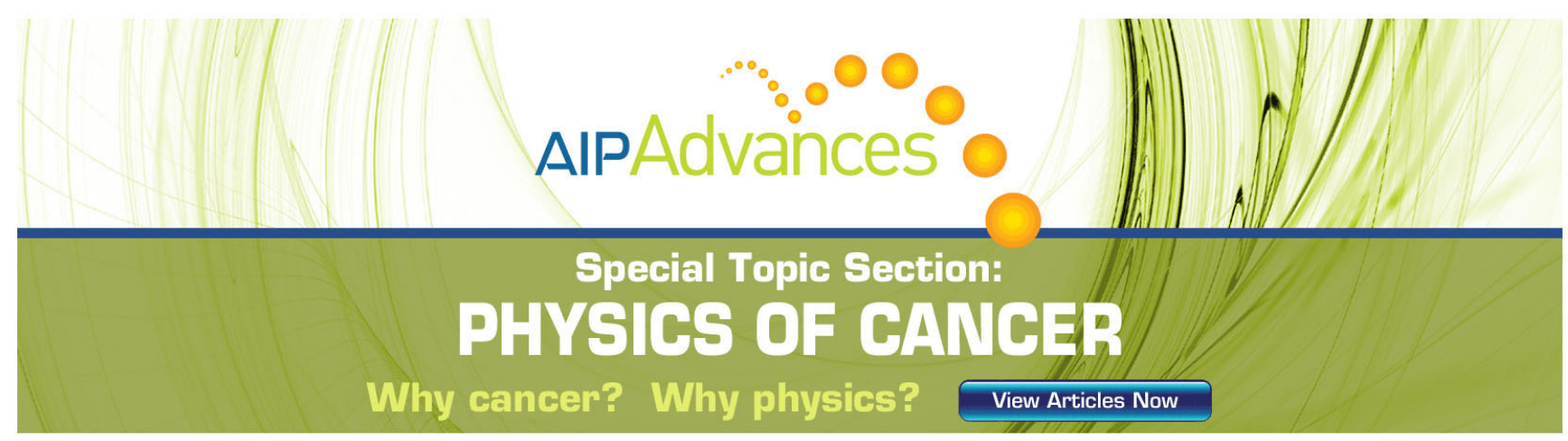




\title{
A 2D simulation study of Langmuir, whistler, and cyclotron maser instabilities induced by an electron ring-beam distribution
}

\author{
K. H. Lee, ${ }^{1}$ Y. Omura, ${ }^{2}$ and L. C. Lee ${ }^{1}$ \\ ${ }^{1}$ Institute of Space Science, National Central University, Taoyuan, Taiwan \\ ${ }^{2}$ Research Institute for Sustainable Humanosphere, Kyoto University, Uji, Kyoto, Japan
}

(Received 10 May 2011; accepted 29 July 2011; published online 16 September 2011)

We carried out a series of 2D simulations to study the beam instability and cyclotron maser instability (CMI) with the initial condition that a population of tenuous energetic electrons with a ring-beam distribution is present in a magnetized background plasma. In this paper, weakly relativistic cases are discussed with the ring-beam kinetic energy ranging from 25 to $100 \mathrm{keV}$. The beam component leads to the two-stream or beam instability at an earlier stage, and the beam mode is coupled with Langmuir or whistler mode, leading to excitation of beam-Langmuir or beamwhistler waves. When the beam velocity is large with a strong beam instability, the initial ring-beam distribution is diffused in the parallel direction rapidly. The diffused distribution may still support $\mathrm{CMI}$ to amplify the $\mathrm{X} 1$ mode (the fundamental $\mathrm{X}$ mode). On the contrary, when the beam velocity is small and the beam instability is weak, CMI can amplify the Z1 (the fundamental Z mode) effectively while the $\mathrm{O} 1$ (the fundamental $\mathrm{O}$ mode) and $\mathrm{X} 2$ (the second harmonic $\mathrm{X}$ mode) modes are very weak and the $\mathrm{X} 1$ mode is not excited. In this report, different cases with various parameters are presented and discussed for a comprehensive understanding of ring-beam instabilities. (C) 2011 American Institute of Physics. [doi:10.1063/1.3626562]

\section{INTRODUCTION}

The cyclotron maser instability $(\mathrm{CMI})$ is an important mechanism for radio emission processes in plasma physics and astrophysics. Wu and Lee, ${ }^{1}$ Lee and $\mathrm{Wu}^{2}{ }^{2}$ and Lee $e t a l .{ }^{3}$ first applied CMI to the emission mechanism of auroral kilometric radiation (AKR). ${ }^{4-6}$ They predicted the growth of $\mathrm{X}$ and $\mathrm{O}$ modes while previous studies on CMI were about only the $\mathrm{Z}$ mode. ${ }^{7-9}$ Wagner et al ${ }^{10,11}$ verified the amplification of $\mathrm{X}$ and $\mathrm{O}$ modes by carrying out a series of particle simulations with a loss-cone distribution function. The key ingredients for CMI are the relativistic effect on the resonance condition, resonance ellipses in the momentum space, and a populationinversion distribution function providing free energy. According to these characteristics, there are various kinds of distribution functions that can support CMI. ${ }^{12-54}$ Pritchett $^{18-21}$ and Strangeway ${ }^{22,23}$ studied CMI with ring-like and DGH distribution functions. Pritchett et $a l^{27,28}$ simulated CMI with a horse-shoe distribution function, which was recently identified as being more similar to the true distribution function in the AKR source region and compared the horse-shoe model with observations.

As the cyclotron maser theory was extensively discussed, the ring-beam problem drew the attention of scientists. Freund et $a{ }^{12}{ }^{12}$ introduced a ring-beam distribution and studied the associated CMI. $\mathrm{Wu}^{13}$ and Leroy and Mangeney ${ }^{14}$ proposed a possible scenario for the formation of a ring-beam distribution in quasi-perpendicular shocks. Wu et al. ${ }^{47}$ and Yoon et $a l^{48}$ studied CMI with the assumption of ring-beam distribution formed by mirror reflection of an injection of a plasma beam accelerated by magnetic reconnection in the low corona. Many literatures on the ring-beam CMI were published. ${ }^{34-51}$ Applications to various types of solar radio bursts and to radiations of astrophysical shocks were also studied. ${ }^{44-50}$ It is believed that a ring-beam distribution can provide an effective radiation source for radio emissions. Kainer et al. ${ }^{39,45}$ and Lee et $a l{ }^{43}$ studied the ring-beam CMI via 1-D simulations with $\omega_{p e} / \Omega_{c e}>1$ and $\omega_{p e} / \Omega_{c e}=0.2$, respectively, where $\omega_{p e} / \Omega_{c e}$ is the ratio of electron plasma to electron cyclotron frequency. The value of $\omega_{p e} / \Omega_{c e}$ is an important parameter for CMI, and it is usually much less than unity in regions of strong CMI.

So far, the ring-beam instabilities in most literatures were studied via kinetic theories or 1D simulations. However, by means of such methods, one has assumed that all waves propagate in a specific direction. Obviously, the ringbeam problem requires a $2 \mathrm{D}$ solver because the two major instabilities, CMI and the two-stream or beam instability, in such a system take place in very different directions. The CMI waves mainly propagate in the perpendicular and quasiperpendicular directions, and those of beam instability are in the parallel and quasi-parallel directions. In order to study the ring-beam problem, we carried out a series of 2D simulations. The considered kinetic energy ranges from several to $100 \mathrm{keV}$, although the low-energy $(\leq 10 \mathrm{keV})$ cases do not show clear wave amplification and thus are not presented. In this report, we mainly focus on physics of wave amplification rather than applications to specific problems.

In the following, we first introduce the simulation model and then generally discuss the simulation results. Three cases are studied in detail as examples. In the Sec. IV, a summary of simulation results is given.

\section{SIMULATION MODEL}

We use a 2D electromagnetic particle-in-cell simulation code modified from the $1 \mathrm{D}$ one, KEMPO1, ${ }^{55,56}$ to study the ring-beam problem. The simulation box lies in the $x-y$ 
plane. Periodic boundaries are applied in both directions. In the initial condition, all particles are uniformly distributed in the simulation box with a constant ambient magnetic field $\mathbf{B}_{\mathbf{0}}=B_{0} \hat{x}$. The protons are assumed to be cold and immobile. Particle distributions are studied in a momentum space rather than a non-relativistic velocity space, and the term "momentum" shall refer to "momentum per unit mass" or "relativistic velocity u". A ring-beam distribution function in the cylindrical coordinates can be expressed as

$$
f_{r b}=n_{r b} a_{r b} \exp \left[-\frac{\left(u_{\perp}-u_{d \perp}\right)^{2}+\left(u_{\|}-u_{d \|}\right)^{2}}{2(\Delta u)^{2}}\right]
$$

where $u_{\perp}$ and $u_{\|}$are the momentum components perpendicular and parallel to $\mathbf{B}_{\mathbf{0}}$, respectively; $u_{d \perp}$ and $u_{d \|}$ are the ringbeam momenta perpendicular and parallel to $\mathbf{B}_{\mathbf{0}}$, respectively; $\Delta u$ is a momentum dispersion of a ring-beam distribution; $a_{r b}$ is a normalization constant. The density of a ringbeam distribution $n_{r b}$ is taken to be $5 \%$ of total electron density. The background electrons are assumed to possess a Maxwellian distribution with a thermal momentum equal to $0.05 c$, and the momentum dispersion of a ring-beam distribution is $\Delta u=0.025 c$, where $c$ is the speed of light. Here, we define a parameter $\phi_{p}=\tan ^{-1}\left(u_{d \perp} / u_{d \|}\right)$ as the average pitch angle of a ring-beam distribution. A schematic illustration of a ring-beam distribution as well as background electrons is drawn in Fig. 1. In the simulations, the primary parameters used are (1) the ratio of electron plasma to electron cyclotron frequency $\omega_{p e} / \Omega_{c e}=0.2$, (2) the average pitch angle $\phi_{p}=15,30,45,60,75$, and 90, and (3) the Lorentz factor $\gamma=\sqrt{1+\left(u_{d \perp}^{2}+u_{d \|}^{2}\right) / c^{2}}=1.05,1.1$, and 1.2. The average initial kinetic energy of ring-beam electrons with $\gamma=1.05$, 1.1 , and 1.2 corresponds to 25,50 , and $100 \mathrm{keV}$, respectively. Only these weakly relativistic cases are considered. Other cases with $\gamma \leq 1.02(10 \mathrm{keV})$ do not show clear wave

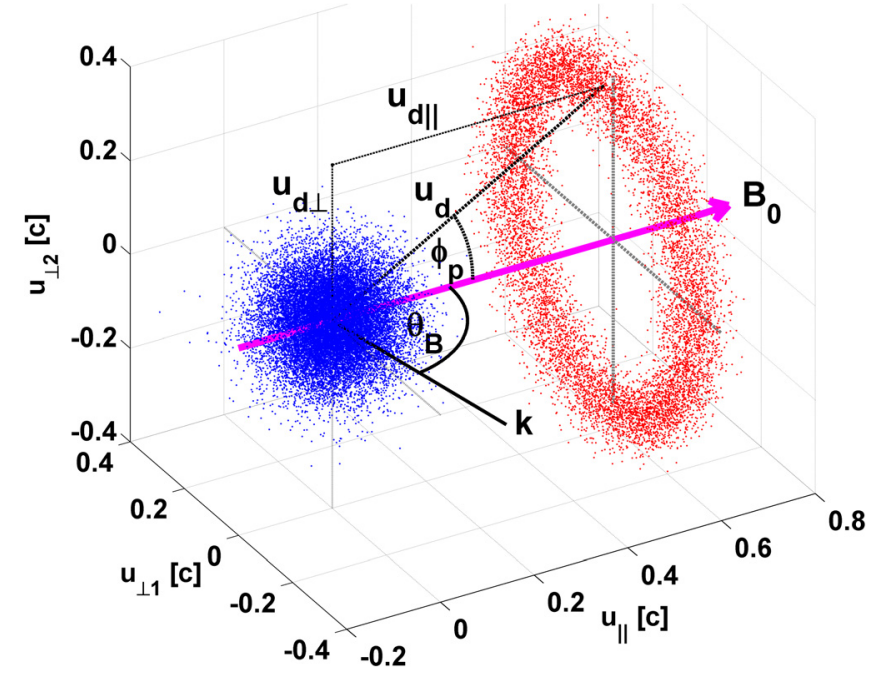

FIG. 1. (Color online) A schematic illustration of a ring-beam distribution (red) as well as background electrons (blue) in the 3D momentum space, where $u_{d}=\sqrt{u_{d \perp}^{2}+u_{d \|}^{2}}$ is a ring-beam momentum and $\theta_{B}$ is a wave propagation angle. In the $3 \mathrm{D}$ momentum space, $u_{\perp 1}, u_{\perp 2}$, and $u_{\|}$are three orthogonal axes, where $u_{\|}$is parallel to $\mathbf{B}_{\mathbf{0}}$. amplification and thus are not discussed here. Note that, in the following discussion, a harmonic number refers to the absolute value of number $n$ in the resonance condition, $k_{\|} u_{\|}+n \Omega_{c e}=\omega \gamma$, where $\omega$ is a wave angular frequency; $k_{\|}$ and $k_{\perp}$ are the parallel and perpendicular components of wave vector $\mathbf{k}$, respectively.

\section{SIMULATION RESULTS}

In Figs. 2(a)-2(d), the diagrams show the maximum $E_{x}$, $E_{y}, E_{z}$, and $B_{z}$ wave amplitude in $\omega$ domain as a function of $k_{\perp}$ and $k_{\|}$in different cases, where $E_{x}$ is the electric component parallel to $\mathbf{B}_{\mathbf{0}} ; B_{z}$ and $E_{z}$ are purely transverse components. In Fig. 2(a), $\left(E_{x}\right)$, we can find intensive excitations of beam-Langmuir (B-L) mode in the parallel direction and beam-whistler (B-W) mode in oblique directions in cases with $\phi_{p} \leq 45$, and the first harmonic $\mathrm{O}(\mathrm{O} 1)$ mode can be observed with low intensity in cases with $\phi_{p}=45,60$, and 75. In Fig. 2(b), $\left(E_{y}\right)$, we can find intensive excitations of beam-whistler mode in oblique directions in cases with $\phi_{p} \leq 45$; the first harmonic $\mathrm{Z}$ (Z1) and second harmonic $\mathrm{X}$ (X2) modes can also be observed in cases with $\phi_{p} \geq 60$. In Fig. 2(c), $\left(E_{z}\right), \mathrm{Z} 1$ and X2 modes can be found mainly in cases with $\phi_{p} \geq 60$, and the first harmonic $\mathrm{X}(\mathrm{X} 1)$ mode is found in cases with $\phi_{p}=30$ and 45. In Fig. 2(d), $\left(B_{z}\right)$, we can find the beam-whistler mode in cases with $\phi_{p} \leq 45$ and the $\mathrm{O}$ mode in cases with $\phi_{p}=45,60$, and 75 .

Table I summarizes the unstable modes in cases with different energies $(\gamma=1.05,1.1$, and 1.2) and different initial pitch angles $\left(\phi_{p}=15,30,45,60,75\right.$, and 90). Generally, we have CMI and beam instability in ring-beam plasma. The beam momentum leads to the beam instability exciting the beam-whistler and beam-Langmuir modes as in cases with $\phi_{p}=15$ and 30 . The ring momentum can support CMI amplifying the $\mathrm{Z}, \mathrm{X}$, and $\mathrm{O}$ modes as in cases with $\phi_{p}=75$ and 90 . The cases with $\phi_{p}=45$ and 60 are transitional ones showing the competition between beam instability and CMI.

In the following, we use different simulation cases to illustrate the generation of various wave modes: (1) beamwhistler and beam-Langmuir modes, (2) X1 mode, and (3) $\mathrm{Z} 1, \mathrm{X} 2$, and $\mathrm{O} 1$ modes. Wave modes can be distinguished via different ways. The beam-Langmuir waves propagate mainly in the direction parallel to $\mathbf{B}_{\mathbf{0}}$ with strong ES energy $\left(E_{x}\right)$, and the beam-whistler waves propagate in directions oblique to $\mathbf{B}_{\mathbf{0}}$ with strong ES energy $\left(E_{x}\right.$ and $\left.E_{y}\right)$ and weak EM components $\left(B_{z}\right)$. The Z1 and O1 modes have phase velocities slower and faster than $c$, respectively. For O-mode waves with perpendicular propagation, the electric field is parallel to $\mathbf{B}_{\mathbf{0}}\left(E_{x}\right)$, and the magnetic field is perpendicular to both $\mathbf{B}_{\mathbf{0}}$ and $\mathbf{k}\left(B_{z}\right)$. The electric field of extraordinary ( $\mathrm{Z}$ and $\mathrm{X})$ mode with perpendicular propagation is in the plane perpendicular to $\mathbf{B}_{\mathbf{0}}\left(E_{y}\right.$ and $\left.E_{z}\right)$, and moreover, $E_{z}$ is usually stronger than $E_{y}$.

1. Case A: $\gamma=1.1(50 \mathrm{keV})$ and $\phi_{p}=45$

Figs. 3(a) and 3(b) show the $\omega-k$ analyses with $\theta_{B}=0$ and 45 for case $\mathrm{A}$, in which the Langmuir and whistler modes are shown as eigen-modes on the left, and the mode couplings between beam and Langmuir modes and between beam and whistler modes are shown on the right. 

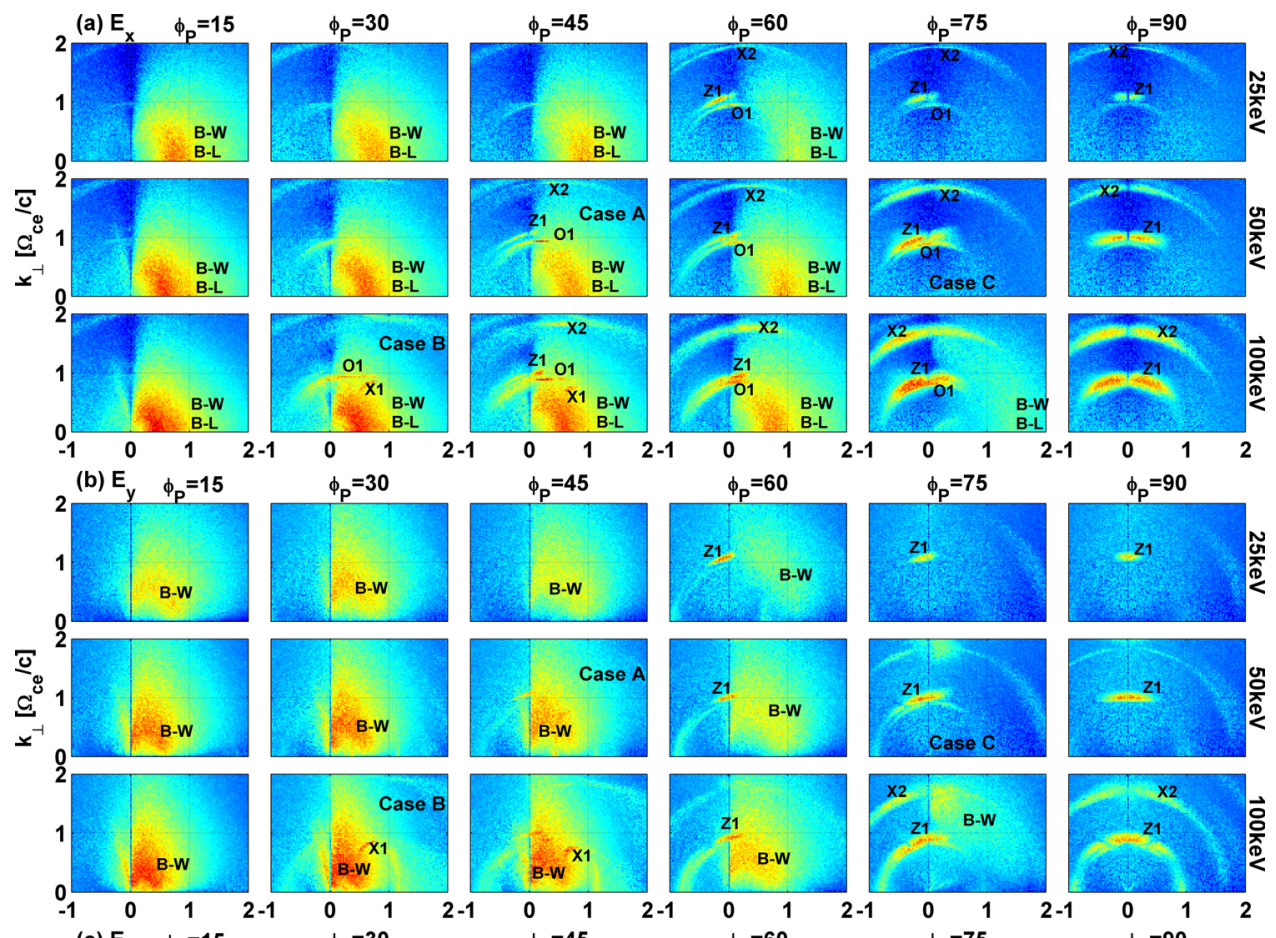

FIG. 2. (Color online) Maximum (a) $E_{x}$ wave amplitude, (b) $E_{y}$ wave amplitude, (c) $E_{z}$ wave amplitude, and (d) $B_{z}$ wave amplitude in $\omega$ domain as a function of $k_{\perp}$ and $k_{\|}$in different cases. Wave modes, X1, X2, O1, Z1, B-L, and B-W, are designated in the panels; $\mathrm{B}-\mathrm{W}$ and $\mathrm{B}-\mathrm{L}$ refer to the beam-whistler and beam-Langmuir modes, respectively. The color bar is shown at the bottom, and all panels have the same contour scale with the normalization $20 \log _{10}\left[\left(E_{x}, E_{y}, E_{z}, c B_{z}\right) / c B_{0}\right]$. Cases A, B, and $\mathrm{C}$ are labeled in the corre-

sponding panels.
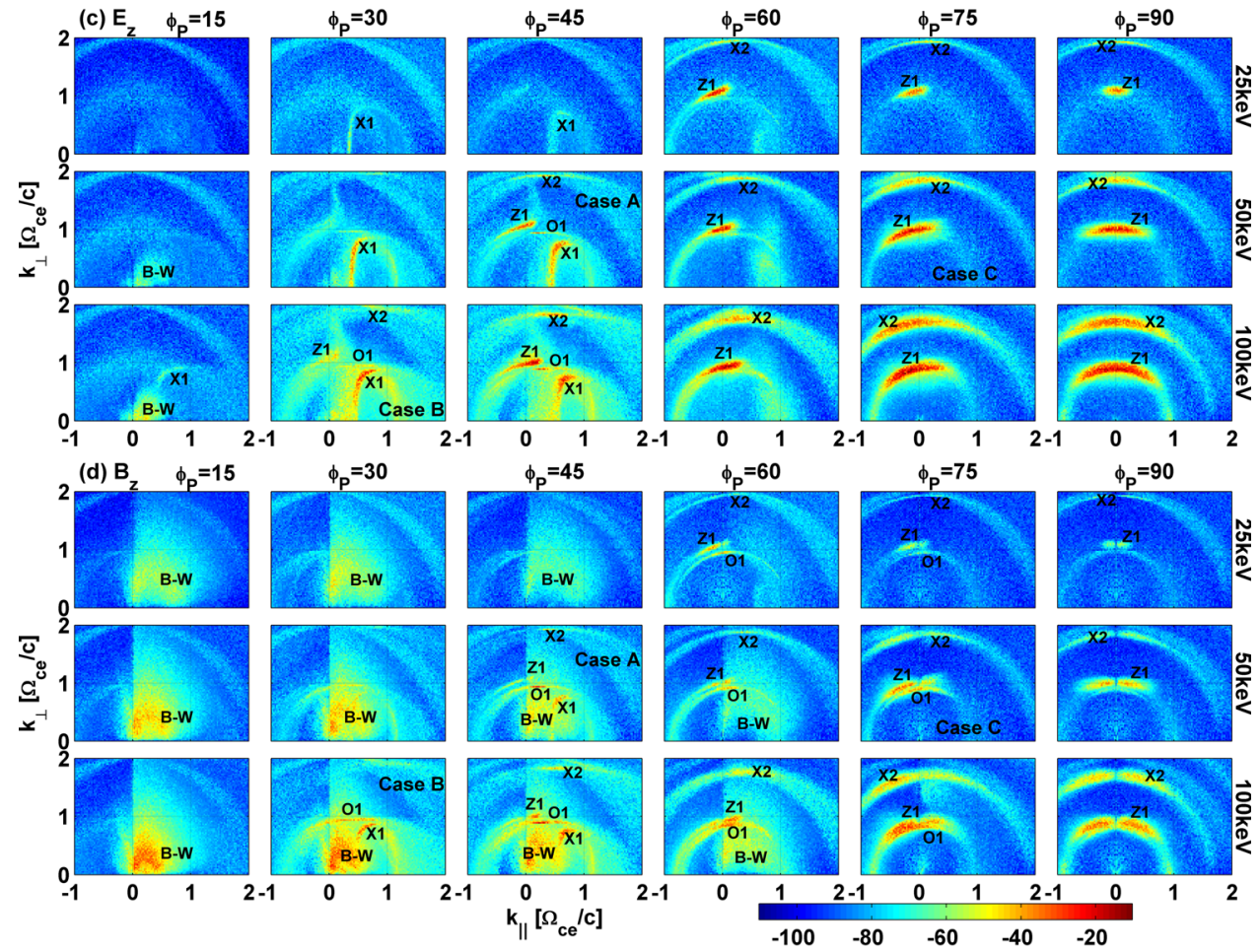

In Fig. 3(a), the Langmuir mode on the right couples with the beam mode causing the formation of two branches of coupled modes in the dispersion diagram. The beam component leads to the excitation of beam-Langmuir (B-L) mode. The waves of lower branch are much more intensive. In Fig. 3(b), the whistler mode on the right couples with the beam mode resulting in the formation of two branches in the dispersion diagram. The beam component leads to the excitation of beam-whistler (B-W) mode. The waves of lower branch are more intensive. Figs. 3(c) and 3(d) show maximum wave amplitude of different components in $\omega$ domain as a function of $k_{\perp}$ and $k_{\|}$. We can see an overall wave amplification of beam-Langmuir and beam-whistler modes in $k_{\perp}-k_{\|}$space, and the beamwhistler mode can be excited over a wide range of $\theta_{B}$. The amplification of other wave modes will be discussed in other cases.

2. Case B: $\gamma=1.2(100 \mathrm{keV})$ and $\phi_{p}=30$

Figs. 4(a)-4(d) show the $\omega-k$ analyses with given $\theta_{B}$, and Figs. 4(e)-4(h) show maximum wave amplitude of different components in $\omega$ domain as a function of $k_{\perp}$ and $k_{\|}$. In case $\mathrm{B}$, we can also find excitations of beam-Langmuir 
TABLE I. A list of unstable wave modes in different cases with different $\gamma$ and $\phi_{p}$. B-W and B-L refer to the beam-whistler and beam-Langmuir modes, respectively; $\mathrm{X} 1, \mathrm{Z} 1$ and $\mathrm{O} 1$ represent the first harmonic $\mathrm{X}, \mathrm{Z}$, and $\mathrm{O}$ modes, respectively; $\mathrm{X} 2$ is the second harmonic $\mathrm{X}$ mode. In each case, the wave modes are listed in the sequence of their peak energies, and those listed in parallel have comparable peak energies. Case A $(\gamma=1.1$ with $\left.\phi_{p}=45\right)$, case B $\left(\gamma=1.2\right.$ with $\left.\phi_{p}=30\right)$, and case C $(\gamma=1.1$ with $\left.\phi_{p}=75\right)$ are discussed in detail as examples.

\begin{tabular}{|c|c|c|c|c|c|c|}
\hline$\gamma \quad \phi_{p}$ & 15 & 30 & 45 & 60 & 75 & 90 \\
\hline \multirow{3}{*}{$\begin{array}{l}1.05 \\
(25 \mathrm{keV})\end{array}$} & B-W & B-W & B-W & $\mathrm{Z} 1$ & $\mathrm{Z} 1$ & $\mathrm{Z} 1$ \\
\hline & B-L & B-L & B-L & B-W/O1 & $\mathrm{X} 2$ & $\mathrm{X} 2$ \\
\hline & & $\mathrm{X} 1$ & $\mathrm{X} 1$ & $\mathrm{X} 2$ & $\mathrm{O} 1$ & \\
\hline \multirow{3}{*}{$\begin{array}{l}1.1 \\
(50 \mathrm{keV})\end{array}$} & B-W & B-W & B-W & $\mathrm{Z} 1$ & $\mathrm{Z} 1$ & $\mathrm{Z} 1$ \\
\hline & B-L & B-L & B-L & B-W & $\mathrm{X} 2 / \mathrm{O} 1$ & $\mathrm{X} 2$ \\
\hline & & $\mathrm{X} 1$ & $\mathrm{O} 1 / \mathrm{Z} 1 / \mathrm{X} 1$ & $\mathrm{~B}-\mathrm{L} / \mathrm{O} 1 / \mathrm{X} 2$ & & \\
\hline \multirow{4}{*}{$\begin{array}{l}1.2 \\
(100 \mathrm{keV})\end{array}$} & B-W & B-W & B-W & $\mathrm{Z} 1$ & $\mathrm{Z1}$ & $\mathrm{Z} 1$ \\
\hline & B-L & B-L & B-L & B-W & $\mathrm{X} 2 / \mathrm{O} 1$ & $\mathrm{X} 2$ \\
\hline & & $\mathrm{X} 1$ & $\mathrm{Z} 1$ & B-L & & \\
\hline & & & $\mathrm{X} 1 / \mathrm{O} 1$ & $\mathrm{X} 2 / \mathrm{O} 1$ & & \\
\hline
\end{tabular}

[Fig. 4(a)] and beam-whistler modes [Figs. 4(b) and 4(d)] as in case A but with much higher intensity due to the higher beam momentum of ring-beam electrons. The presence of $B_{z}$ component of beam-whistler mode implies that it is an EM mode, and its ES component is stronger than its EM component. Fig. 4(i) shows the energy histories of particles and different wave modes. The energy of beamwhistler mode is higher than that of beam-Langmuir mode. The loss of total kinetic energy is about $10 \%$ at the saturation of beam modes, and most of lost kinetic energy is transferred to the beam-whistler and beam-Langmuir modes.

Case B also shows strong amplification of X1 mode. In Fig. 4(g), the intensive part forming a small arc is the X1 mode. The Z1 mode in Figs. 4(c) and 4(g) is faint, and the $\mathrm{O} 1$ mode can be found with low intensity in Figs. 4(e) and 4(h). The X1-mode energy, in this case, is about $0.2 \%$ of initial total kinetic energy at its saturation as can be seen in Fig. 4(i). The amplified X1 mode is close to the $\mathrm{X}$-mode cut-off frequency. These waves are very dispersive as shown in Figs. 4(b)-4(d). In addition, the group velocity varies from near zero at $k \approx 0$ to the speed of light at $c k / \Omega_{c e} \approx 1$. The observed frequency-time spectrum should have fine structures showing a falling tone.

Fig. 5 shows the momentum distribution functions of energetic electrons of case B at different times. The first panel shows the initial distribution, and the second one corresponds to the time after the saturation of beam-whistler and beam-Langmuir modes. Since the X1-mode amplification occurs around and after the saturation of beam modes [Fig. 4(i)], the amplification of X1 mode in this case is mainly supported by the deformed distribution in Fig. 5(b). In Fig. 5(c), corresponding to the time after saturation of the growth of X1 mode, we can find a strong diffusion of energetic electrons in the direction toward $u_{\perp}=0$, and the diffusive pattern can be related to the resonance ellipses $\left(k_{\|} \boldsymbol{u}_{\|}+n \boldsymbol{\Omega}_{c e}=\omega \gamma\right)$ in momentum space. The waves corresponding to these resonance ellipses are all X1-mode waves, and the electrons around these ellipses resonate with the waves. Energy transfer between electrons and waves occurs via wave-particle resonance and leads to the diffusive pattern in Fig. 5(c). Due to the highly dispersive curve of X1 mode in Figs. 4(b)-4(d) and the X1-mode cut-off frequency higher than the cyclotron frequency, a large beam velocity is needed to satisfy both the first harmonic cyclotron resonance condition $\left(k_{\|} u_{\|}+\Omega_{c e}=\omega \gamma\right)$ and the X-mode dispersion relation for the X1-mode excitation. In other words, for the amplification of $\mathrm{X} 1$ mode via the ring-beam CMI, a ring momentum provides free energy, and a beam momentum brings electrons into the resonant domain.

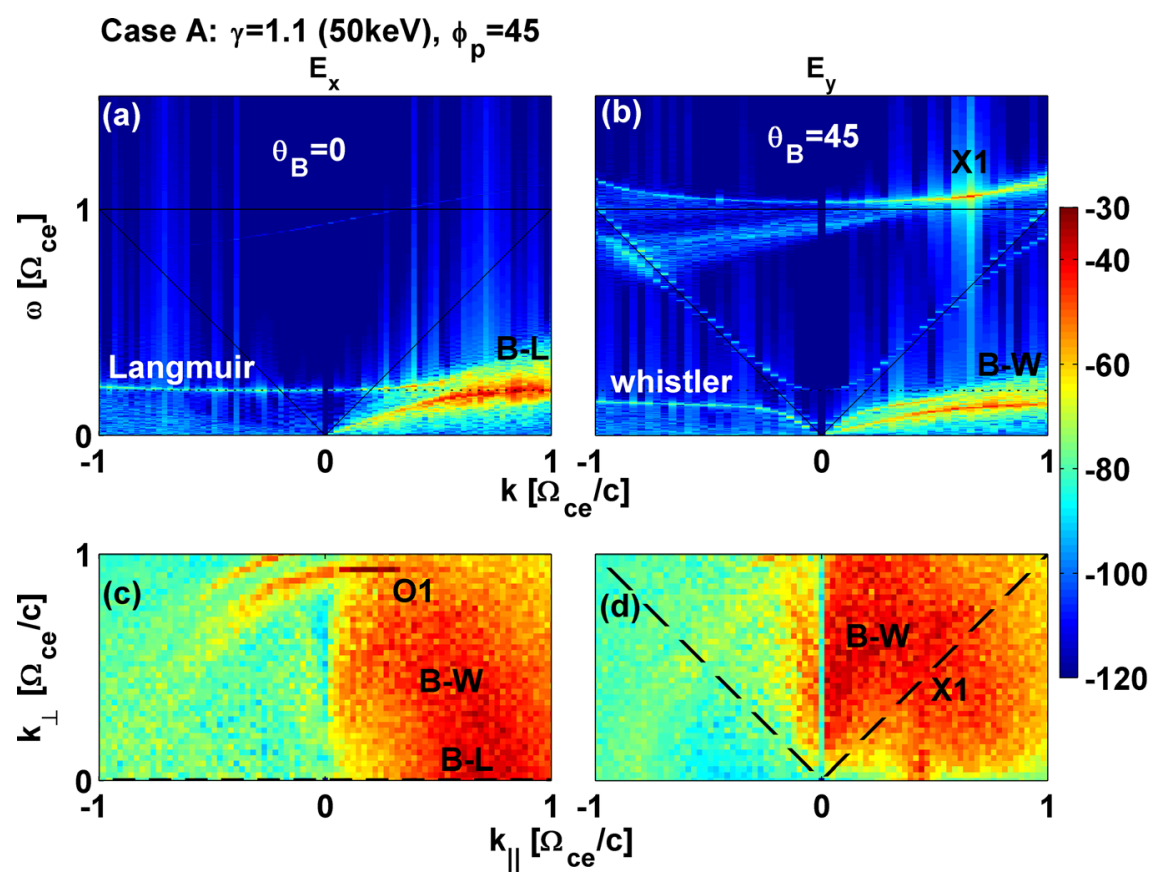

FIG. 3. (Color online) Diagrams associated with case A: (a) (b) $\omega-k$ spectra with given $\theta_{B}$, and (c) (d) maximum wave amplitude of different components in $\omega$ domain as a function of $k_{\perp}$ and $k_{\|}$. In panels (a) and (b), the slope of V-like lines is the speed of light. In panels (c) and (d), the dashed lines designate the directions of $\theta_{B}$ of $\omega-k$ analyses. All panels have the same contour scale with same normalization as that of Fig. 2 for different wave components, and the color bar is shown on the right. Note that the contour scale is shifted compared with Fig. 2 so that the color contour is enhanced. 
Case B: $\gamma=1.2(100 \mathrm{keV}), \phi_{p}=30$
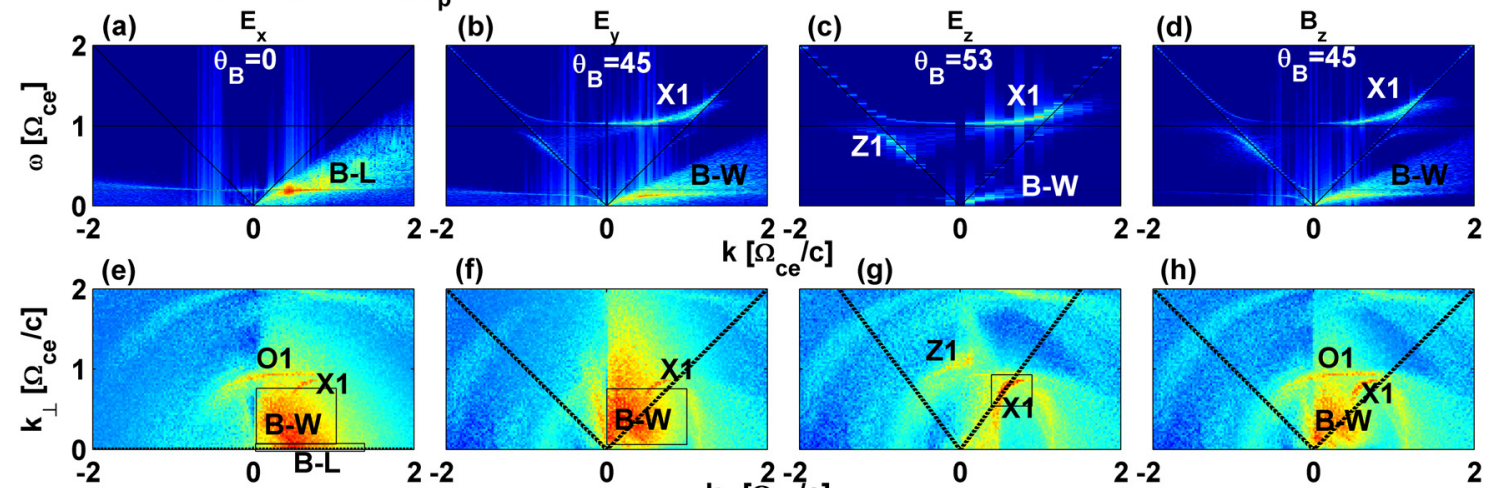

(g)

(h)
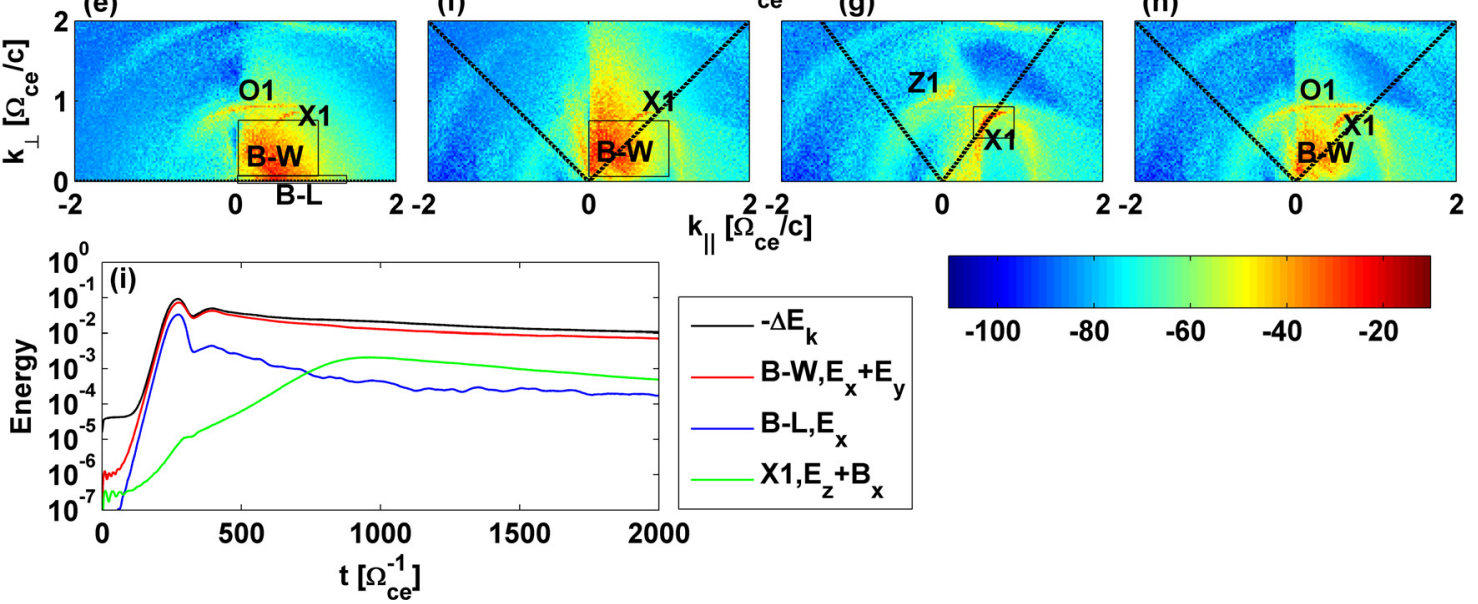

FIG. 4. (Color online) Diagrams associated with case B: (a) (b) (c) (d) $\omega-k$ spectra of different components with given $\theta_{B}$; (e) (f) (g) (h) the same diagrams as those labeled case B in Fig. 2; (i) time history of decrement of total kinetic energy ( $-\Delta E_{k}$ ), and energy histories of different wave modes. In panels (a), (b), (c), and (d), the slope of V-like lines is the speed of light. In panels (e), (f), (g), and (h), the dashed lines designate the directions of $\theta_{B}$ of $\omega-k$ analyses, and the rectangles designate the range of $k_{\perp}$ and $k_{\|}$of waves corresponding to the wave energy histories. The color bar is shown at the bottom, and all panels have the same contour scale with same normalization as that of Fig. 2 for different wave components. The energy histories are normalized to the initial total energy.
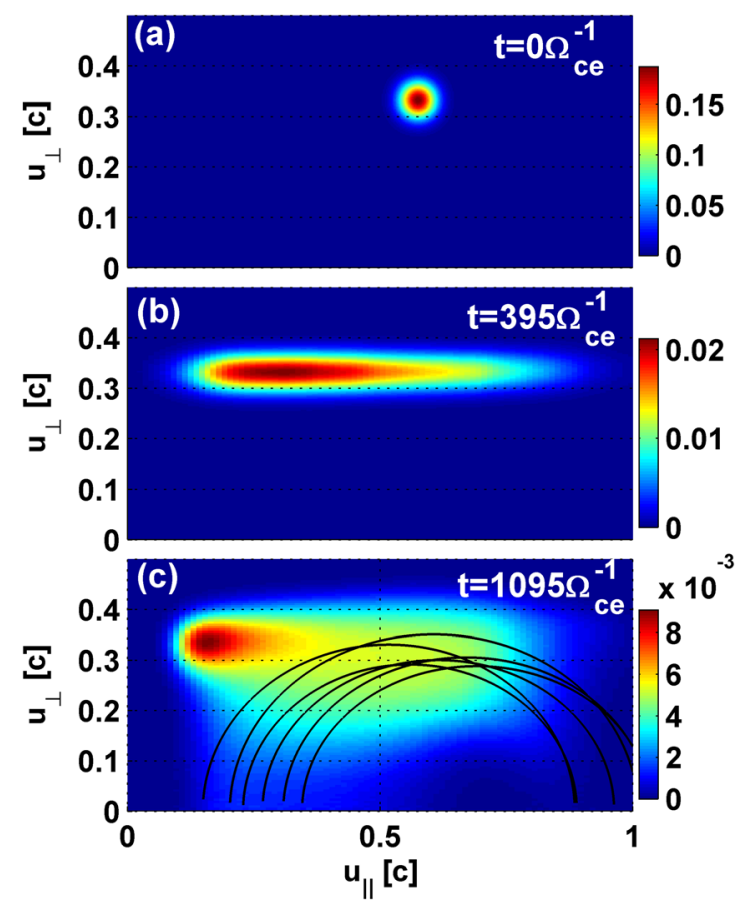

FIG. 5. (Color online) Momentum distribution functions $f\left(u_{\perp}, u_{\|}\right)$of energetic electrons of case B at (a) $t=0$, (b) $t=395 \Omega_{c e}^{-1}$, and (c) $t=1095 \Omega_{c e}^{-1}$, corresponding to the initial condition, the time after the saturation of beamwhistler and beam-Langmuir modes, and after the saturation of X1 mode, respectively. In panel (c), the resonance ellipses of waves with large $E_{z}$ found in the spectra of case B are plotted.
3. Case $\mathrm{C}: \gamma=1.1(50 \mathrm{keV})$ and $\phi_{p}=75$

Figs. 6(a)-6(d) show the $\omega-k$ analyses with $\theta_{B}=90$, and Figs. 6(e)-6(h) show maximum wave amplitude of different components in $\omega$ domain as a function of $k_{\perp}$ and $k_{\|}$. Fig. 6(i) shows the energy histories of particles and different wave modes. In case $\mathrm{C}$, the $\mathrm{Z} 1$ mode is dominant as shown in Fig. 6(i) and can be found with strong intensity in Figs. 6(c) and 6(g). However, the Z1 mode cannot propagate into a field-free region without mode conversion, ${ }^{51-54}$ which could highly decrease the emission efficiency. Since we consider a uniform simulation system, the mode conversion is not discussed here. The X2 and $\mathrm{O} 1$ modes can be found in Figs. 6(c) and 6(g) and Figs. 6(a)-6(h), respectively. They have wave intensities of the same order of magnitude and are much weaker than the $\mathrm{Z} 1$ mode [Fig. 6(i)]. These Z1-, X2-, and O1-mode waves propagate mainly in the perpendicular and quasi-perpendicular directions. In Fig. 6(i), the loss of total kinetic energy at the end of the simulation is about $4.7 \%$; the $\mathrm{Z1} 1-\mathrm{X} 2-$, and $\mathrm{O} 1-$ mode energies are about $4.6 \%, 0.06 \%$, and $0.03 \%$ of initial total kinetic energy, respectively. For overtone modes, only the X2 mode can be observed, while the $\mathrm{O} 2$ mode is too weak to be of importance.

Fig. 7 shows the momentum distribution functions of energetic electrons of case $\mathrm{C}$ at different times. Figs. 7(a) 7 (c) correspond to the initial condition, the time after the saturation of $\mathrm{Z} 1, \mathrm{X} 2$, and $\mathrm{O} 1$ modes, and the last time step of simulation, respectively. Since the initial ring momentum is much larger than the beam momentum, a vigorous CMI and a strong diffusion of ring-beam distribution in the direction towards $u_{\perp}=0$ as shown in Figs. 7(b) and 7(c) are expected 

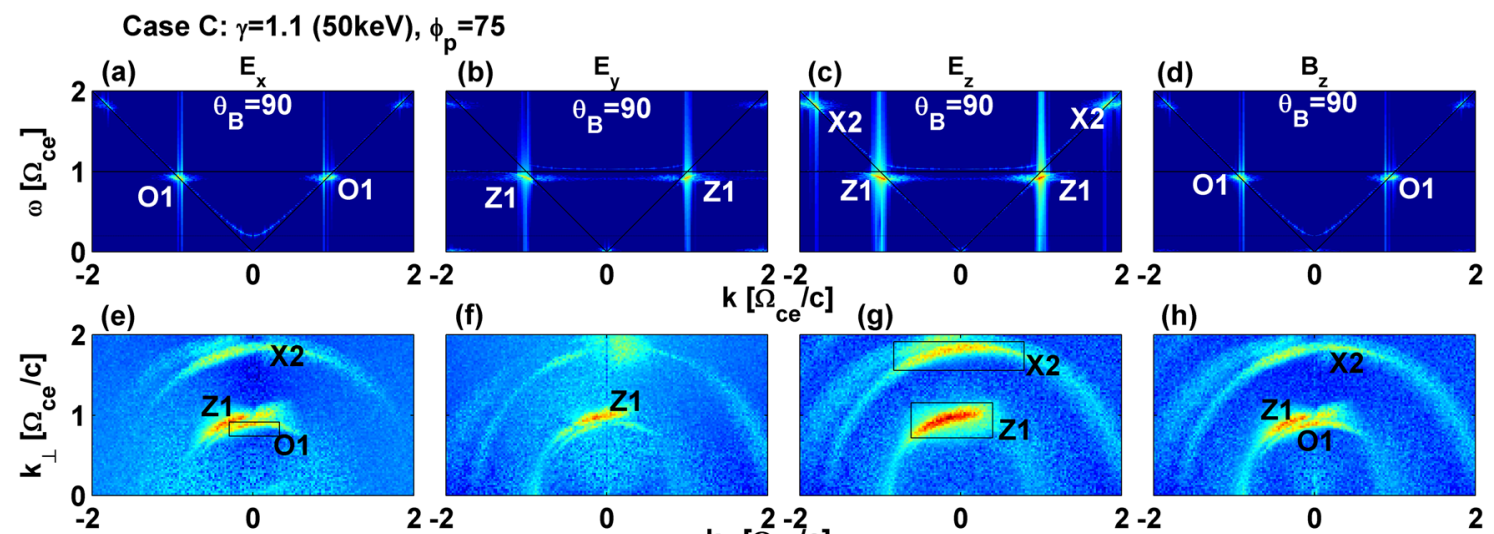

(g)

(h)
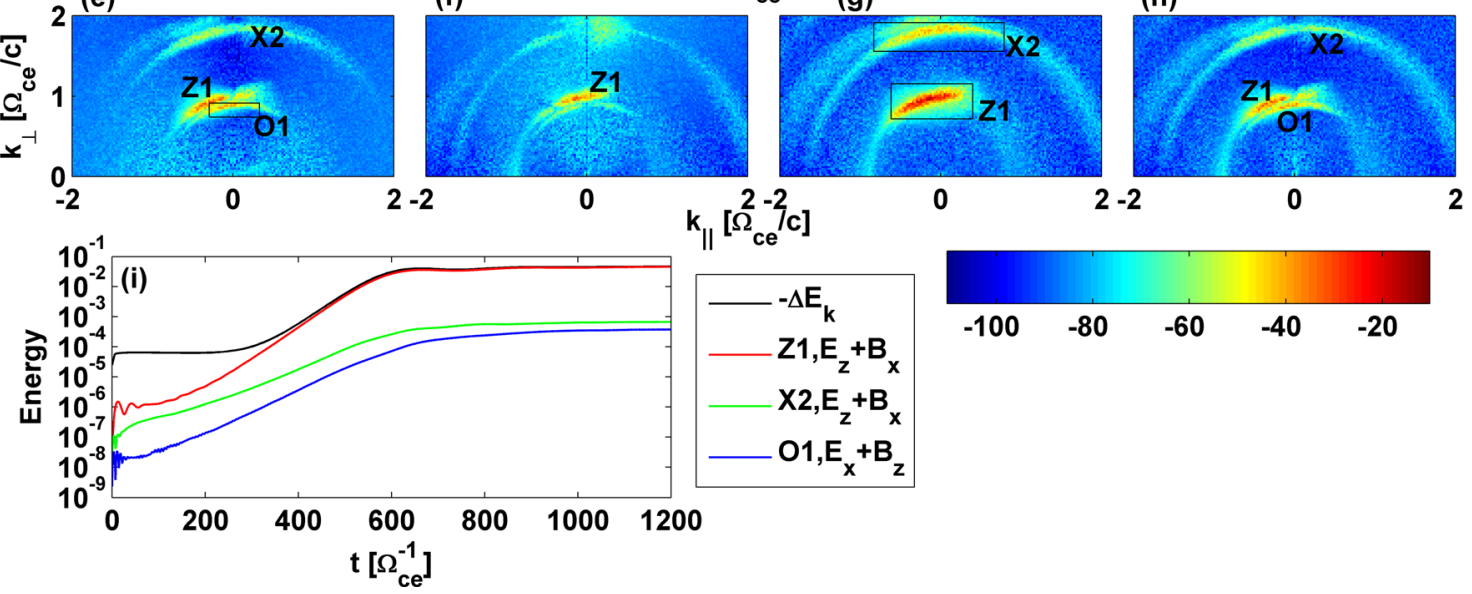

FIG. 6. (Color online) Diagrams associated with case C: (a) (b) (c) (d) $\omega-k$ spectra of different components with $\theta_{B}=90$, (e) (f) (g) (h) the same diagrams as those labeled case $\mathrm{C}$ in Fig. 2, (i) time history of decrement of total kinetic energy ( $-\Delta E_{k}$ ) and energy histories of different wave modes. In panels (a), (b), (c), and (d), the slope of V-like lines is the speed of light. In panels (e), (f), (g), and (h), the rectangles designate the range of $k_{\perp}$ and $k_{\|}$of waves corresponding to the wave energy histories. The color bar is shown at the bottom, and all panels have the same contour scale with same normalization as that of Fig. 2 for different wave components. The energy histories are normalized to the initial total energy.

from the resonance conditions associated with the kinetic theory of CMI. The loss of perpendicular kinetic energy contributes mostly to the Z1-mode waves. In Fig. 7(c), the resonance ellipses $\left(k_{\|} u_{\|}+n \Omega_{c e}=\omega \gamma\right)$ in momentum space are plotted, and the corresponding waves are all Z1-mode waves. The diffusive pattern of energetic electron distribution is related to these resonance ellipses. Energy transfer between electrons and waves occurs via wave-particle resonance and leads to the diffusive pattern in Figs. 7(b) and 7(c).

\section{SUMMARY}

We carried out a series of 2D simulations to study CMI with the initial condition that a tenuous ring-beam distribu- tion is present in magnetized plasma with $\omega_{p e} / \Omega_{c e}=0.2$. Table I shows the unstable wave modes in different cases. The simulation results can be summarized as following. (1) The beam component of a ring-beam distribution excites the ES beam-Langmuir and EM beam-whistler modes, and these modes dominate in cases with $\phi_{p} \leq 45$. (2) For the amplification of $\mathrm{X} 1$ mode via the ring-beam CMI, a ring momentum provides free energy and a large beam momentum brings electrons into the resonant domain. (3) In cases with $\phi_{p} \geq 60$, the Z1 mode dominates and is amplified effectively via CMI due to a large ring momentum while the X2 mode is relatively weak. (4) The O1 mode can be observed in cases with $\phi_{p}=45,60$, and 75, although its intensity is not high.

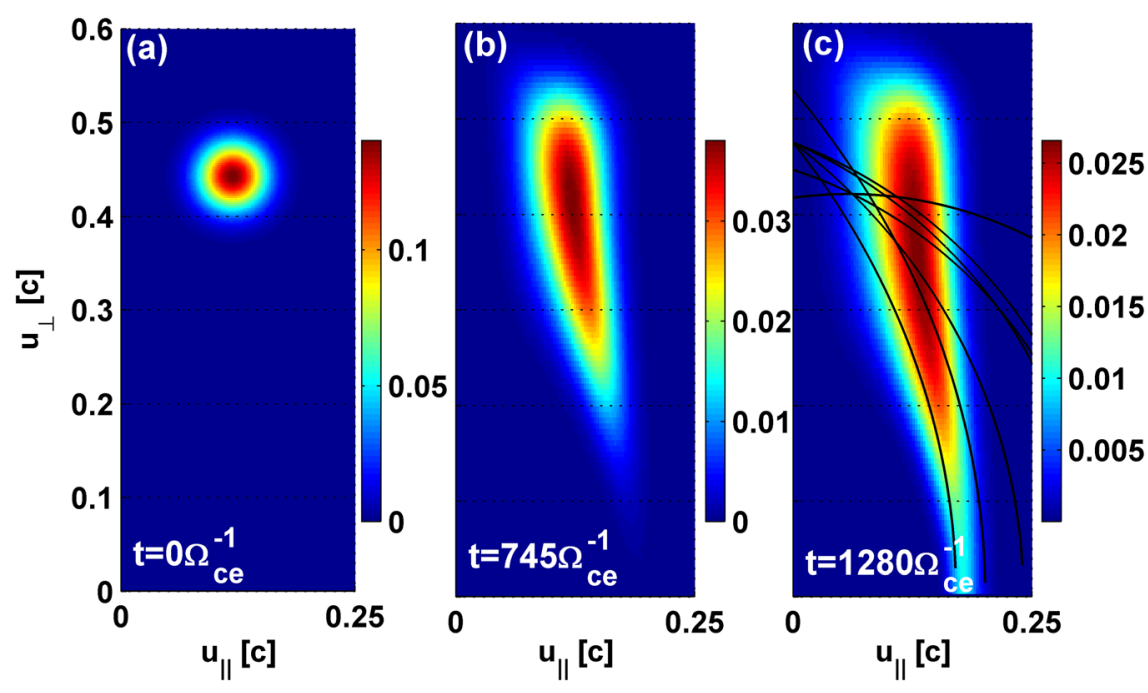

FIG. 7. (Color online) Momentum distribution functions $f\left(u_{\perp}, u_{\|}\right)$of energetic electrons of case $\mathrm{C}$ at (a) $t=0$, (b) $t=745 \Omega_{c e}^{-1}$, and (c) $t=1280 \Omega_{c e}^{-1}$, corresponding to the initial condition, the time after the saturation of CMI modes, and the last time step of simulation, respectively. In panel (c), the resonance ellipses of waves with large $E_{z}$ found in the spectra of case $\mathrm{C}$ are plotted. 
In previous studies of ring-beam CMI based on linear theory or $1 \mathrm{D}$ simulations, it was shown that the $\mathrm{O}$ mode could also be amplified with a high growth rate. ${ }^{41-43}$ However, our simulation result is different from the previous ones. The $\mathrm{O} 1$ mode can only be found with low intensity in cases with $\phi_{p}=45,60$, and 75, and the $\mathrm{O} 2$ mode is too weak to be of importance.

Finally, let us briefly discuss the difference between the planetary radio emission model and our ring-beam model. In the studies of AKR and other planetary kilometric radiations originating from polar regions, the density of energetic electrons is assumed to be dominant. ${ }^{17,18,20-23}$ In those cases, the linear dispersion relations in the plasma are affected by the energetic electrons, and the relativistic effect may make the $\mathrm{X}$-mode cut-off frequency lower than the relativistic cyclotron frequency, which makes the amplification of X1 mode possible even with zero beam velocity. In our model, however, the density of energetic ring-beam electrons is much smaller than that of background low-energy electrons, and the background electrons determine the eigen-modes of plasma while the ringbeam electrons control the resonance excitation. In such a case, for the amplification of X1 mode, a large beam momentum is needed to satisfy both the first harmonic cyclotron resonance condition and the $\mathrm{X}$-mode dispersion relation.

In this report, we briefly present the $2 \mathrm{D}$ simulation results associated with our ring-beam model. A further complete study of ring-beam instabilities will be conducted with a strongly relativistic effect and higher values of $\omega_{p e} / \Omega_{c e}$.

\section{ACKNOWLEDGMENTS}

This work was partially supported by Grant-in-Aid 20340135 and 17 GS0208 of the Ministry of Education, Science, Sports, and Culture of Japan and partially by the National Science Council in Taiwan under Grant No. NSC97-2111-M-006-001-MY3. We are also grateful to the National Center for High-performance Computing in Taiwan for computer time and facilities.

${ }^{1}$ C. S. Wu and L. C. Lee, Astrophys. J. 230, 621 (1979).

${ }^{2}$ L. C. Lee and C. S. Wu, Phys. Fluids 23, 1348 (1980).

${ }^{3}$ L. C. Lee, J. R. Kan, and C. S. Wu, Planet. Space Sci. 28, 703 (1980).

${ }^{4}$ D. A. Gurnett, J. Geophys. Res. 79, 4227, doi:10.1029/ JA079i028p04227(1974).

${ }^{5}$ M. L. Kaiser and R. G. Stone, Science 189, 285 (1975).

${ }^{6}$ R. F. Benson and W. Calvert, Geophys. Res. Lett. 6, 479, doi:10.1029/ GL006i006p00479 (1979)

${ }^{7}$ R. Q. Twiss, Asutral. J. Phys. 11, 564 (1958).

${ }^{8}$ J. Schneider, Phys. Rev. Lett. 2, 504 (1959).

${ }^{9}$ H. P. Freund and C. S. Wu, Phys. Fluids 20, 619 (1977).

${ }^{10}$ J. S. Wagner, T. Tajima, L. C. Lee, and C. S. Wu, Geophys. Res. Lett. 10, 483, doi:10.1029/GL010i006p00483 (1983).

${ }^{11}$ J. S. Wagner, L. C. Lee, C. S. Wu, and T. Tajima, Radio Sci. 19, 509, doi: 10.1029/RS019i002p00509 (1984).

${ }^{12}$ H. P. Freund, H. K. Wong, C. S. Wu, and M. J. Xu, Phys. Fluids 26, 2263 (1983).

${ }^{13}$ C. S. Wu, J. Geophys. Res. 89, 8857, doi:10.1029/JA089iA10p08857 (1984).

${ }^{14}$ M. M. Leroy and A. Mangeney, Ann. Geophys. 2, 449 (1984).

${ }^{15}$ L. C. Lee, "Theories of non-thermal radiations from planets," in Plasma Waves and Instabilities at Comets and in Magnetospheres (A90-49901 23-75) (American Geophysical Union, Washington, DC, 1989), p. 239.
${ }^{16}$ C. S. Wu, C. S. Lin, H. K. Wong, S. T. Tsai, and R. L. Zhou, Phys. Fluids 24, 2191 (1981).

${ }^{17}$ R. M. Winglee, Phys. Plasmas 25, 217 (1983).

${ }^{18}$ P. L. Pritchett, J. Geophys. Res. 89, 8957, doi:10.1029/JA089iA10p08957 (1984).

${ }^{19}$ P. L. Pritchett, Phys. Fluids 27, 2393 (1984).

${ }^{20}$ P. L. Pritchett, Geophys. Res. Lett. 11, 143, doi:10.1029/GL011i002p00143 (1984).

${ }^{21}$ P. L. Pritchett, Phys. Fluids 29, 2919 (1986).

${ }^{22}$ R. J. Strangeway, J. Geophys. Res. 90, 9675, doi:10.1029/JA090iA10p09675 (1985).

${ }^{23}$ R. J. Strangeway, J. Geophys. Res. 91, 3152, doi:10.1029/JA091iA03p03152 (1986).

${ }^{24}$ H. K. Wong, C. S. Wu, and J. JD Gaffey, Phys. Fluids 28, 2751 (1985).

${ }^{25}$ P. Louarn, D. Quéau, and A. Roux, Solar Phys. 111, 201 (1987).

${ }^{26}$ P. H. Yoon and L. F. Ziebell, Phys. Rev. E 51, 4908 (1995).

${ }^{27}$ P. L. Pritchett, R. J. Strangeway, C. W. Carlson, R. E. Ergun, J. P. McFadden, and G. T. Delory, JGR 104, 10317, doi:10.1029/ 1998JA900179 (1999).

${ }^{28}$ P. L. Pritchett, R. J. Strangeway, R. E. Ergun, and C. W. Carlson, J. Geophys. Res. 107, 1437, doi:10.1029/2002JA009403 (2002).

${ }^{29}$ R. E. Ergun, C. W. Carlson, J. P. McFadden, G. T. Delory, R. J. Strangeway, and P. L. Pritchett, Astrophys. J. 538, 456 (2000).

${ }^{30}$ R. Bingham and R. A. Cairns, Phys. Plasmas 7, 3089 (2000).

${ }^{31}$ R. Bingham, B. J. Kellett, R. A. Cairns, J. Tonge, and J. T. Mendonça, Astrophys. J. 595, 279 (2003).

${ }^{32}$ I. Vorgul, R. A. Cairns, and R. Bingham, Phys. Plasmas 12, 122903 (2005).

${ }^{33}$ T. Umeda, M. Ashour-Abdalla, D. Schriver, R. L. Richard, and F. V. Coroniti, J. Geophys. Res. 112, A04212, doi:10.1029/2006JA012124 (2007).

${ }^{34}$ C. S. Wu and H. P. Freund, Radio Sci. 19, 519, doi:10.1029/ RS019i002p00519 (1984).

${ }^{35}$ C. S. Wu, G. C. Zhou, and J. J. D. Gaffey, Phys. Fluids 28, 846 (1985).

${ }^{36}$ C. S. Wu, Space Sci. Rev. 41, 215 (1985).

${ }^{37}$ B. R. Shi, J. J. D. Gaffey, and C. S. Wu, Phys. Fluids 29, 4212 (1986).

${ }^{38}$ H. P. Freund, J. Q. Dong, C. S. Wu, and L. C. Lee, Phys. Fluids 30, 3106 (1987).

${ }^{39}$ S. Kainer, J. J. D. Gaffey, C. P. Price, X. W. Hu, and G. C. Zhou, Phys. Fluids 31, 2238 (1988).

${ }^{40}$ P. H. Yoon, Phys. Fluids B 2, 867 (1990).

${ }^{41}$ Y. P. Chen, G. C. Zhou, P. H. Yoon, and C. S. Wu, Phys. Plasmas 9, 2816 (2002).

${ }^{42}$ P. H. Yoon, C. B. Wang, and C. S. Wu, Phys. Plasmas 14, 022901 (2007).

${ }^{43}$ K. H. Lee, Y. Omura, L. C. Lee, and C. S. Wu, Phys. Rev. Lett. 103, 105101 (2009).

${ }^{44}$ C. S. Wu, R. S. Steinolfson, and G. C. Zhou, Astrophys. J. 309, 392 (1986).

${ }^{45}$ S. Kainer and R. J. MacDowall, J. Geophys. Res. 101, 495, doi:10.1029/95JA02026 (1996).

${ }^{46}$ W. M. Farrell, J. Geophys. Res. 106, 15701, doi:10.1029/2000JA000156 (2001).

${ }^{47}$ C. S. Wu, C. B. Wang, P. H. Yoon, H. N. Zheng, and S. Wang, Astrophys. J. 575, 1094 (2002).

${ }^{48}$ P. H. Yoon, C. S. Wu and C. B. Wang, Astrophys. J. 576, 552 (2002).

${ }^{49}$ C. S. Wu, M. J. Reiner, P. H. Yoon, H. N. Zheng and S. Wang, Astrophys. J. 605, 503 (2004).

${ }^{50}$ C. S. Wu, C. B. Wang, G. C. Zhou, S. Wang and P. H. Yoon, Astrophys. J. 621, 1129 (2005).

${ }^{51}$ A. O. Benz and G. Thejappa, Astron. Astrophys. 202, 267 (1988).

${ }^{52}$ R. M. Winglee and G. A. Dulk, Astrophys. J. 307, 808 (1986).

${ }^{53}$ J. LaBelle, R. A. Treumann, P. H. Yoon and M. Karlicky, Astrophys. J. 593, 1195 (2003).

${ }^{54}$ D. B. Melrose, Astrophys. J. 380, 256 (1991).

${ }^{55}$ Y. Omura and H. Matsumoto, "KEMPO1: Technical guide to one dimensional electromagnetic particle code," in Computer Space Plasma Physics. Simulation Techniques and Software, edited by H. Matsumoto and Y. Omura (Terra Scientific, Tokyo, 1993), pp. 21-65.

${ }^{56}$ Y. Omura, "One-dimensional electromagnetic particle code: KEMPO1," in Advanced Methods for Space Simulations, edited by H. Usui and Y. Omura (Terra Sci., Tokyo, 2007), pp. 1-21. 